\title{
Survival Status and Predictors of Mortality Among Preterm Neonates Admitted to Mizan Tepi University Teaching Hospital, South West Ethiopia
}

\author{
Bayisa Bereka' \\ Tigist Demeke' \\ Belete Fenta iD ${ }^{2}$ \\ Yalemtsehay Dagnaw ${ }^{3}$ \\ 'School of Nursing, Jimma University, \\ Jimma, Oromia Regional State, Ethiopia; \\ ${ }^{2}$ School of Midwifery, Jimma University, \\ Jimma, Oromia Regional State, Ethiopia; \\ ${ }^{3}$ Department of Nursing, Mizan Tepi \\ University, Mizan Tepi, South Nations, \\ Nationalities and People Region, Ethiopia
}

Objective: The main objective of this study was to assess survival status and predictors of mortality among preterm neonates admitted to Mizan Tepi University Teaching Hospital.

Materials and Methods: A retrospective cohort study was conducted on 568 randomly selected neonates. All preterm neonates admitted in NICU from May 9, 2016 to December 30, 2019 were the source population. Data were extracted from medical chart and entered to EpiData version 4.4.2.1 and analyzed by STATA version 14. Descriptive statistics, bivariate and multivariable analyses were done in a Cox regression model. Variables with $P$-value of $<0.05$ were considered statistically significant in predicting the preterm mortality.

Results: Out of 568 preterm neonates, 199 (35\%) died with an incidence rate of 62.15 (54.09-71.41) deaths per 1000 person-day-observations with median survival time of 15 days. Vaginal mode of delivery (adjusted hazard ratio, AHR: 1.78, 95\%CI: 1.05-3.08), noncephalic presentation (AHR:1.8, 95\%CI: 1.04-3.06), born from mothers with no ANC follow-up (AHR: 1.9, 95\%CI: 1.29-3.01), fifth minute APGAR score $<7$ (AHR: 1.87, 95\% CI: 1.31-2.68), RDS (AHR: 1.74, 95\%CI: 1.28-2.36), did not receive KMC (AHR: 1.45, (95\%CI: 1.06-1.98), did not cry immediately after birth (AHR: 2.81, 95\%CI: 2.03-3.93)), VLBW (AHR: 2.67, 95\%CI: 1.29-5.53), low birth weight (AHR: 2.24, 95\%CI: 1.15-4.39), and hypothermia (AHR: 1.36, 95\%CI: 1-1.84) were significantly associated with preterm mortality.

Conclusion: Preterm neonatal mortality was high and the predictors were almost all preventable and treatable. Therefore, emphasis should be given toward prevention and early anticipation, and management of these predictors.

Keywords: censored, mortality, preterm neonate, predictors, survival

\section{Introduction}

According to World Health Organization (WHO), a preterm neonate refers to a baby born before 37 completed weeks of gestation. ${ }^{1}$ Preterm neonates can be further classified into: extremely preterm ( $<28$ weeks), very preterm $(28$ to $<32$ weeks) and moderate preterm (32 to $<37$ weeks) of gestation. ${ }^{1,2}$ Even though prematurity is a global burden, there is survival difference between developing and developed countries. Neonates born in Africa had 12 times higher risk of mortality compared to those born in Europe. ${ }^{3}$ Despite the survival of preterm neonates improving significantly with the advent of highly specialized intensive care, they contribute highly to neonatal deaths in resource-limited settings including 
Ethiopia. ${ }^{4}$ In developing countries, in 2012 prematurityrelated neonatal mortality was the second cause of early neonatal mortality and $83 \%$ of deaths that occurred in the first week of life. ${ }^{5}$ In different regions of Ethiopia, prematurity was the major contributor to neonatal deaths. In Ethiopia in 2019 neonatal mortality rate was reported to be 30 deaths per 1000 live births ${ }^{6}$ and prematurity contributed about $11 \%$ of under-five mortalities. ${ }^{7}$ In Tigray, Northern Ethiopia $34 \%$ of neonatal deaths were attributable to prematurity. ${ }^{8}$ From public hospitals in Eastern Ethiopia, around $28.58 \%$ of neonatal deaths were due to preterm complications. ${ }^{9}$ In Mizan Tepi University Teaching Hospital (MTUTH), from all neonatal deaths $(22.8 \%)$, more than two-thirds $(76 \%)$ of deaths were caused by prematurity. $^{10}$

The sustainable development goal three emphasizes reducing neonatal deaths with a goal of 12 neonatal deaths per 1000 live births per country by 2030 through different interventions. ${ }^{11}$ Despite this sustainable development goal and many efforts, in many lower middle-income countries including Ethiopia, there is increased prevalence of preterm birth and high neonatal mortality. Furthermore, in previous studies, even though prematurity was the foremost cause of neonatal mortality, ${ }^{10}$ survival status of preterm neonates and predictors of mortality is not well cited. In addition, the survival rate and predictors of preterm mortality may be varied from place-to-place and time-totime due to different factors. To develop and modify the existing interventional packages and guidelines, it is difficult to utilize single/few study finding/s to intervene rather than conducting a systematic review. Therefore, this study will have a great contribution to reach the existing evidence and missed evidence. This result of this study will be a source for further study and systematic review that will further have input in policy developing and care planning. This study result will ultimately have its implication in the reduction of preterm mortality. Therefore, assessing the survival status and identifying the predictors of mortality are extremely essential.

\section{Materials and Methods}

\section{Study Design and Setting}

An institution-based retrospective cohort study was conducted among preterm neonates admitted to the neonatal intensive-care unit (NICU) of Mizan Tepi University Teaching Hospital (MTUTH). Data were collected during March to April 2020. The hospital is located $584 \mathrm{~km}$ from
Addis Ababa in Southwest Ethiopia. The hospital serves a population of more than two million people. The hospital started to give NICU service from May 8, 2016. The NICU had 17 beds, and 11 nurses were working in the unit. In the NICU of the hospital, 963 preterm neonates were admitted from May 9, 2016 to December 30, 2019. The data were collected during March to April 2020.

\section{Study Participants}

All preterm neonates admitted in the NICU from May 9, 2016 to December 30, 2019 were recruited as source population using the admission registration book. Then, a sequential number was assigned for each preterm neonate's medical card. A simple random sampling technique was used to select the desired sample size by using computer-generated random numbers. The preterm neonates whose charts were incomplete on major variables (gestational age, date of admission, date of death/censored) and lost cards were excluded. From selected medical cards, 20 and 29 were incomplete and lost cards, respectively. These charts were replaced with new randomly selected cards.

\section{Data Collection Tool and Methods}

The checklist for this study was adapted from a similar study conducted in Tikur Anbessa specialized hospital ${ }^{12}$ and modified after reviewing of other related literature. ${ }^{13,14}$ A checklist consisted of the information on maternal and neonatal sociodemographic factors, neonatal related factors, preterm related complication/comorbidity factors, maternal medical and obstetric-related factors. The data were extracted from each individual neonate's medical card using a structured checklist. Since the data were collected from neonates' medical card, to decrease misinterpretation of data, the data collectors were trained on how to extract appropriate data and there was close daily monitoring and supervision at the data collection site. For a single neonate's medical card, there were two data collectors and they cross-checked the data collected by one data collector by comparing with the information on the medical card.

\section{Sample Size Determination}

The required sample size was determined by using STATA statistical package version 14 (Cox model) by considering the following assumptions: hazard ratio (HR) (1.55) of the selected covariate (perinatal asphyxia) and probability of failure (event) 0.288 were taken from a previous study. ${ }^{13}$ Variability $(\mathrm{SD})=0.5$ was used for covariates of interest., 
Probability of failure (event) of 0.252 was taken from a previous study. ${ }^{15}$ In addition, 5\% margin of error, 95\% confidence interval and $80 \%$ power was used to calculate the sample size. Perinatal asphyxia was selected out of 12 covariates because it gave a maximum sample. According to the above assumptions, the desired sample size was 568 .

\section{Data Analysis and Statistical Methods}

The data were coded and entered to EpiData statistical software package version 4.4.2.1. Then, the data were exported to STATA version 14 and analyzed. Descriptive statistics was carried out to see the distribution of study variables. Kaplan-Meier anlysis was used to estimate median survival time, cumulative probability of survival, and compare survival difference between the different covariates. Log rank test was also used to compare statistical survival difference between categories of different explanatory variables. Life table was used to estimate the cumulative probability of survival at the different time intervals. Multicollinearity of each independent variable was checked using variance inflation factor (VIF) and mean VIF became 1.17 which shows no collinearity among variables. Cox regression model was used to identify the independent predictors of preterm mortality. Those variables having $P$-value $<0.25$ in the bivariate analysis were transferred to the multivariable analysis and those variables having $P$-value $<0.05$ at $95 \%$ confidence level were considered as independent predictors of preterm mortality.

\section{Operational Definitions}

Survival status: is the outcome of a premature neonate; either death or censored.

Censored: preterm neonates those alive beyond 28 days of a neonatal period, those left against medical advice, discharged with improvement and referred to other health institutions were considered as censored.

\section{Ethical Consideration}

To conduct this study, the authors tried to address the Declaration of Helsinki Ethical principles for medical research. In this study, it was impossible to get informed consent from the neonates' family because the authors had no contact either with the neonates or families and the data were collected from neonates' medical chart after they discharged. But, the study was conducted after approval of a research ethics committee. Ethical clearance with reference number of IRB00016/2020 was obtained from Institutional Review Board of Institute of Health, Jimma University. A formal letter from Institute of health was taken and submitted to MTUTH to get permission and cooperation. Permission was obtained from the clinical director of the study hospital. Any neonatal personal identification was not utilized in the study. All data were kept strictly confidential and used only for the study purpose.

\section{Results}

In this study, 568 preterm neonates' medical cards were reviewed. They had different lengths of hospital stay with minimum of one day, and a maximum of 28 days with a median follow-up the period of four days, which gave overall 3202 person-days of observation.

\section{Sociodemographic Related Factors}

Almost all (97.71\%) preterm neonates were in the age category of $\leq 7$ days during admission. About 489 $(86.09 \%)$ of mothers were in the age range of 20-34 years with a median age of 26 years. The remaining 45 (7.92\%) and 34 (5.99\%) were found between the group of $\geq 35$ and $\leq 20$ years respectively. Three hundred and ninety (68.66\%) mothers were from rural residency.

\section{Neonatal Related Factors}

Three hundred and fifty-four (62.32\%), 311 (54.75\%), 196 (51.02\%), 173 (30.46\%), 86 (15.14\%), and 64 (11.27\%) of neonates were diagnosed with sepsis, hypothermia, RDS, hypoglycemia, perinatal asphyxia, and jaundice, respectively (Table 1).

Five hundred and fifty $(96.83 \%)$ of the preterm neonates were born at health institutions and 387 (68.13\%) preterm neonates were born with low birth weight (1500$2499 \mathrm{~g}$ ) with a median weight of $1900.00 \mathrm{~g}$. Three hundred and forty-four $(60.56 \%)$ preterm neonates were born with GA of 32-36 weeks with a median GA of 34 weeks. Four hundred and nine (72.01\%) preterm neonates had a first minute APGAR score of $<7$ (Table 2).

\section{Maternal Medicals and Obstetrics Related Factors}

In this study, 89 (15.67\%) preterm neonates were born from mothers who had obstetric complications. Sixty-two $(10.92 \%)$ preterm neonates were born from mothers who had pregnancy-induced hypertension. The majority $(87.50 \%)$ of preterm neonates were delivered through 
Table I Cross Tabulation Neonatal Diagnosis and Neonatal Outcome at NICU of MTUTH, 2020 (N=568)

\begin{tabular}{|c|c|c|c|c|}
\hline Characteristics & Category & Total N (\%) & Death N (\%) & Censored N (\%) \\
\hline \multirow[t]{2}{*}{ Sepsis } & No & $214(37.68)$ & $136(36.45)$ & $78(63.55)$ \\
\hline & Yes & $354(62.32)$ & $233(65.82)$ & $121(34.18)$ \\
\hline \multirow[t]{2}{*}{ Respiratory distress syndrome } & No & $372(65.49)$ & $273(73.39)$ & $99(26.61)$ \\
\hline & Yes & $196(51.02)$ & $96(48.98)$ & $100(51.02)$ \\
\hline \multirow[t]{2}{*}{ Necrotizing enterocolitis } & No & $556(97.89)$ & $367(66.01)$ & $189(33.99)$ \\
\hline & Yes & $12(2.11)$ & $2(16.67)$ & $10(83.33)$ \\
\hline \multirow[t]{2}{*}{ Perinatal asphyxia } & No & $482(84.86)$ & $324(67.22)$ & $158(32.78)$ \\
\hline & Yes & $86(15.14)$ & $45(52.33)$ & $41(47.67)$ \\
\hline \multirow[t]{2}{*}{ Congenital anomalies } & No & $547(96.30)$ & 354 (64.72) & $193(35.28)$ \\
\hline & Yes & $21(3.70)$ & $15(7 \mid .43)$ & $6(28.57)$ \\
\hline \multirow[t]{2}{*}{$\mathrm{INH}$} & No & $562(98.94)$ & $368(65.48)$ & $194(34.52)$ \\
\hline & Yes & $6(1.06)$ & $I(16.67)$ & $5(83.33)$ \\
\hline \multirow[t]{2}{*}{ Jaundice } & No & $504(88.73)$ & $320(63.49)$ & $184(36.54)$ \\
\hline & Yes & $64(11.27)$ & $49(76.56)$ & $15(23.44)$ \\
\hline \multirow[t]{2}{*}{ Pulmonary hypertension } & No & $566(99.65)$ & 367 (64.84) & $199(35.16)$ \\
\hline & Yes & $2(0.35)$ & $2(100)$ & - \\
\hline \multirow[t]{2}{*}{ Hypoglycemia } & No & $393(69.54)$ & $262(66.33)$ & $133(33.67)$ \\
\hline & Yes & $173(30.46)$ & $107(61.85)$ & $66(38.15)$ \\
\hline \multirow[t]{2}{*}{ Hypothermia } & No & $257(45.25)$ & 175 (68.09) & $82(31.91)$ \\
\hline & Yes & $311(54.75)$ & $194(62.38)$ & 117 (37.62) \\
\hline \multirow[t]{2}{*}{ Others $^{a}$} & No & $543(95.6)$ & $352(64.83)$ & 191 (35.17) \\
\hline & Yes & $25(4.4)$ & $17(68)$ & $8(40)$ \\
\hline
\end{tabular}

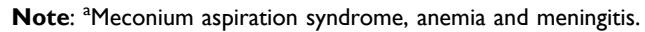

a vaginal mode of delivery and almost more than three quarters $(76.23 \%)$ had a singleton pregnancy (Table 3 ).

\section{Survival Status of Preterm Neonates}

This study showed that 199 (35\%) died with an incidence rate of 62.15 (54.09-71.41) deaths per 1000 person-dayobservations with overall median survival time of 15 days during the period of follow-up and 369 (65\%) were censored of which $273(48.1 \%)$ were discharged with improvement, 71 (12.5\%) left against medical advice, 12 $(2.1 \%)$ transferred to other institutions and the remaining $13(2.3 \%)$ were alive until the end of 28 days. The Kaplan-Meier survival estimate graph shows median survival time (50\%) cumulative survival functions of preterm neonates. At time $t=0$, the estimated probability of continued survival is 1.0. The two perpendicular lines indicate at what time point half of the events occurred which is 15 days. Using the Kaplan-Meier estimator of survival function in this study, the highest; 68 (34.2\%) proportions of mortality occurred on first day the of follow-up period. Moreover, the cumulative probability of survival at the end of 1, 7, and 28-day period of follow-up were $88.03 \%$ (95\% CI: $85-90), \quad 59.37 \% \quad(95 \% \mathrm{CI}: \quad 54-64), \quad 35.90 \% \quad(95 \%$ CI:22.3-49.12) respectively. Using Life table estimator of cumulative failure function, $38.30 \%(95 \% \mathrm{CI}$ : $33.97-$ 42.98) of neonatal deaths occurred within the first 7-day period of follow-up (Figure 1).

\section{Predictors of Preterm Neonatal Mortality}

In this study, preterm neonates diagnosed with RDS were at increased risk of mortality compared to their counterparts. Preterm neonates with a fifth-minute APGAR score of less than seven had higher hazard of mortality compared to those who had a better fifth-minute APGAR score. The hazard ratio of mortality was higher among preterm neonates born with birth weight of 1000-1499 
Table 2 Cross-tabulation of Neonatal Related Factors and Outcome at NICU of MTUTH, 2020 (N=568)

\begin{tabular}{|c|c|c|c|c|}
\hline \multirow[t]{2}{*}{ Characteristics } & \multirow[t]{2}{*}{ Category } & \multirow[t]{2}{*}{ Total N (\%) } & \multicolumn{2}{|c|}{ Outcome } \\
\hline & & & Censored N (\%) & Death N (\%) \\
\hline \multirow[t]{2}{*}{ Place of delivery } & Health institution & $550(96.83)$ & $359(65.27)$ & $194(34.73)$ \\
\hline & Home & $18(3.17)$ & $10(55.56)$ & $8(44.44)$ \\
\hline \multirow[t]{4}{*}{ Weight of neonate (in grams) } & $<1000$ & $6(1.06)$ & $2(33.33)$ & $4(66.67)$ \\
\hline & $1000-1499$ & $101(17.78)$ & $40(39.60)$ & $61(60.40)$ \\
\hline & $1500-2499$ & $387(68.13)$ & $264(68.22)$ & $123(31.78)$ \\
\hline & $\geq 2500$ & $74(13.03)$ & $63(85.14)$ & II (I4.86) \\
\hline \multirow[t]{2}{*}{ GA } & 28-32 weeks & $90(\mid 5.85)$ & $37(4 I . I I)$ & 53 (58.89) \\
\hline & 32-36 weeks & $478(84.15)$ & $332(69.46)$ & $146(30.54)$ \\
\hline \multirow[t]{2}{*}{ APGAR score at Ist minute } & $<7$ & $409(72.01)$ & 247 (60.39) & $162(39.6 I)$ \\
\hline & $\geq 7$ & 159 (27.99) & $122(33.06)$ & $37(18.9)$ \\
\hline \multirow[t]{2}{*}{ APGAR score at 5 th minute } & $<7$ & $214(37.68)$ & 98 (45.79) & $116(54.21)$ \\
\hline & $\geq 7$ & $354(62.32)$ & $27 \mid(76.55)$ & $83(23.45)$ \\
\hline \multirow[t]{2}{*}{ KMC } & Yes & $832(50.35)$ & $211(73.78)$ & $75(26.22)$ \\
\hline & No & $282(49.65)$ & $158(56.03)$ & $124(43.97)$ \\
\hline \multirow[t]{3}{*}{ Weight for gestational age } & LGA & $21(3.70)$ & $17(80.95)$ & $4(19.05)$ \\
\hline & AGA & $426(75.0)$ & 297 (69.72) & $129(30.28)$ \\
\hline & SGA & $121(21.30)$ & $55(45.45)$ & $66(54.55)$ \\
\hline \multirow[t]{2}{*}{ Breathing condition at birth } & Cry at birth & $385(67.78)$ & $308(80.0)$ & $77(20.00)$ \\
\hline & Did not cry at birth & $183(32.22)$ & $61(33.33)$ & $122(66.67)$ \\
\hline
\end{tabular}

$\mathrm{g}$ compared to birth weight of $\geq 2500 \mathrm{~g}$. The hazard ratio of mortality was also higher among preterm neonates who did not receive kangaroo mother care (KMC) than their counterparts. The hazard ratios of mortality were higher among preterm neonates who did not cry immediately after birth compared to their counterparts. Preterm neonates born from mothers who did not have antenatal care (ANC) follow-up had higher hazard ratios of death compared to their counterparts. Preterm neonates who were born via a vaginal mode of delivery had higher hazards of mortality as compared to those born through cesarean section delivery. Similarly, preterm neonates who were born via non-cephalic presentation during birth were at increased risk of mortality than their counterparts. Hypothermic neonates are found to be at increased risk of mortality compared to non-hypothermic neonates (Table 4).

\section{Discussion}

In this study, the overall incidence rate of mortality was 62.15 per 1000 person-day observations (95\%CI: 54.171.4).The cumulative probability of survival at the end of
1,7 , and 28 days of follow-up were $88.03 \%, 59.37 \%$, and $35.90 \%$, respectively. The cumulative failure function indicated that $38.30 \%$ of neonatal deaths occurred within the first 7-day period of follow-up.

The incidence rate of mortality in this study is higher than that of a study done in Tikur Anbessa Hospital, where the incidence rate 39.1 per 1000 person-day observations. ${ }^{12}$ In this study, the overall median survival time of preterm neonates was lower than studies conducted in Addis Ababa ${ }^{12}$ and Gondar, ${ }^{15}$ with overall median survival time of 21 days and 28 days respectively. This difference might be due to the difference in quality of service provision between the hospitals. Probably, hospitals in Addis Ababa and Gondar may have more advanced care and quality services than the current study hospital which is a remote hospital. The proportions of preterm death at the end period of follow-up was 35.04\% (95\%CI: 31.2-39.01) and highest (34.2\%) proportions of mortality occurred on the first day of follow-up. This study is in line with the study done at Jimma University Hospital. ${ }^{14}$ But, the current study result is higher than the study done in Iran, ${ }^{16}$ Pakistan, ${ }^{17}$ University of Gondar Hospital, $^{13}$ Gondar Town Hospital, ${ }^{15}$ and Tikur Anbessa 
Table 3 Cross-tabulation of Maternal Health Problem and Neonatal Outcome at NICU of MTUTH, Ethiopia, 2020 (N=568)

\begin{tabular}{|c|c|c|c|c|c|}
\hline \multicolumn{2}{|l|}{ Characteristics } & Category & Total N (\%) & Censored N (\%) & Death N (\%) \\
\hline \multirow{2}{*}{\multicolumn{2}{|c|}{ Maternal chronic medical disease }} & No & 539 (94.89) & $358(66.42)$ & I8I (33.58) \\
\hline & & Yes & $29(5.11)$ & II (37.93) & $18(62.07)$ \\
\hline \multirow{6}{*}{\multicolumn{2}{|c|}{ Maternal chronic medical disease }} & No & $55 I(97.01)$ & $365(66.24)$ & $186(33.76)$ \\
\hline & & Yes & $17(2.99)$ & $4(23.53)$ & $13(76.47)$ \\
\hline & & No & $357(99.82)$ & $368(64.90)$ & $199(35.10)$ \\
\hline & & Yes & I (0.18) & I (I00) & - \\
\hline & & No & $557(98.06)$ & $364(65.35)$ & $193(34.65)$ \\
\hline & & Yes & $13(2.29)$ & $7(53.83)$ & $6(46.154)$ \\
\hline \multirow{2}{*}{\multicolumn{2}{|c|}{ ANC visit status }} & Yes & $515(90.6)$ & $342(66.4 I)$ & 173 (33.59) \\
\hline & & No & $53(9.33)$ & $27(50.94)$ & $26(49.06)$ \\
\hline \multirow{2}{*}{\multicolumn{2}{|c|}{ Obstetric complications }} & No & $479(84.33)$ & $308(64.30)$ & $174(35.70)$ \\
\hline & & Yes & $89(15.67)$ & $6 \mathrm{I}(68.54)$ & $28(31.46)$ \\
\hline \multirow[t]{8}{*}{ Obstetric complications } & \multirow{2}{*}{$\begin{array}{l}\text { Pregnancy induced } \\
\text { hypertension }\end{array}$} & No & $506(89.08)$ & $324(64.03)$ & I82 (35.97) \\
\hline & & Yes & $62(10.92)$ & 75 (72.58) & $17(27.42)$ \\
\hline & \multirow[t]{2}{*}{ Placenta abruption } & No & $550(96.83)$ & $357(64.9)$ & $193(35.09)$ \\
\hline & & Yes & $18(3.17)$ & $12(66.67)$ & $6(33.33)$ \\
\hline & \multirow[t]{2}{*}{ Placenta previa } & No & $563(99.12)$ & $366(65.01)$ & 197 (34.99) \\
\hline & & Yes & $5(0.88)$ & $3(60)$ & $2(40)$ \\
\hline & \multirow[t]{2}{*}{ Others $^{\mathrm{b}}$} & No & $543(95.6)$ & $352(64.83)$ & $191(35.17)$ \\
\hline & & Yes & $25(4.4)$ & $17(68)$ & $8(40)$ \\
\hline \multirow{2}{*}{\multicolumn{2}{|c|}{ Mode of delivery }} & $\mathrm{C} / \mathrm{S}$ & $7 \mid(\mid 2.50)$ & $49(69.01)$ & $22(30.99)$ \\
\hline & & Vaginal & $497(87.50)$ & $320(64.39)$ & $177(35.61)$ \\
\hline \multirow{2}{*}{\multicolumn{2}{|c|}{ Type of pregnancy }} & Single & $433(76.23)$ & $295(68.13)$ & I38 (31.87) \\
\hline & & Multiple & I35 (23.77) & $74(54.8 \mathrm{I})$ & $61(45.19)$ \\
\hline \multirow{2}{*}{\multicolumn{2}{|c|}{ Maternal febrile illness }} & No & $530(93.31)$ & $35 I(66.23)$ & 179 (33.77) \\
\hline & & Yes & $38(6.69)$ & $18(47.37)$ & $20(52.63)$ \\
\hline \multirow{2}{*}{\multicolumn{2}{|c|}{ Type of presentation }} & Cephalic & $532(93.6)$ & $352(66.17)$ & $18(33.83)$ \\
\hline & & Non-cephalic & $36(6.34)$ & $17(47.22)$ & $19(52.78)$ \\
\hline
\end{tabular}

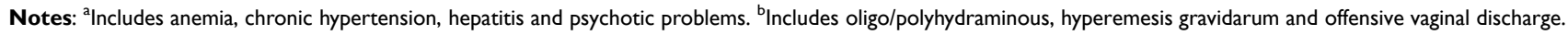

Hospital, ${ }^{12}$ where proportions of death were $9.1 \%, 14 \%$, $28.8 \%, 25.2 \%$, and $29.77 \%$, respectively. The possible reason for the discrepancy with studies done in Iran and Pakistan might be economic and sociodemographic variation between Ethiopia and the two countries. The disparities in economic and sociodemographic variation may in turn lead to disparity in quality of service provided for preterm neonates. In addition, the difference with studies that were done in Gondar and Addis Ababa might be due to variation in the provision of quality service. However, this result is lower than the study 


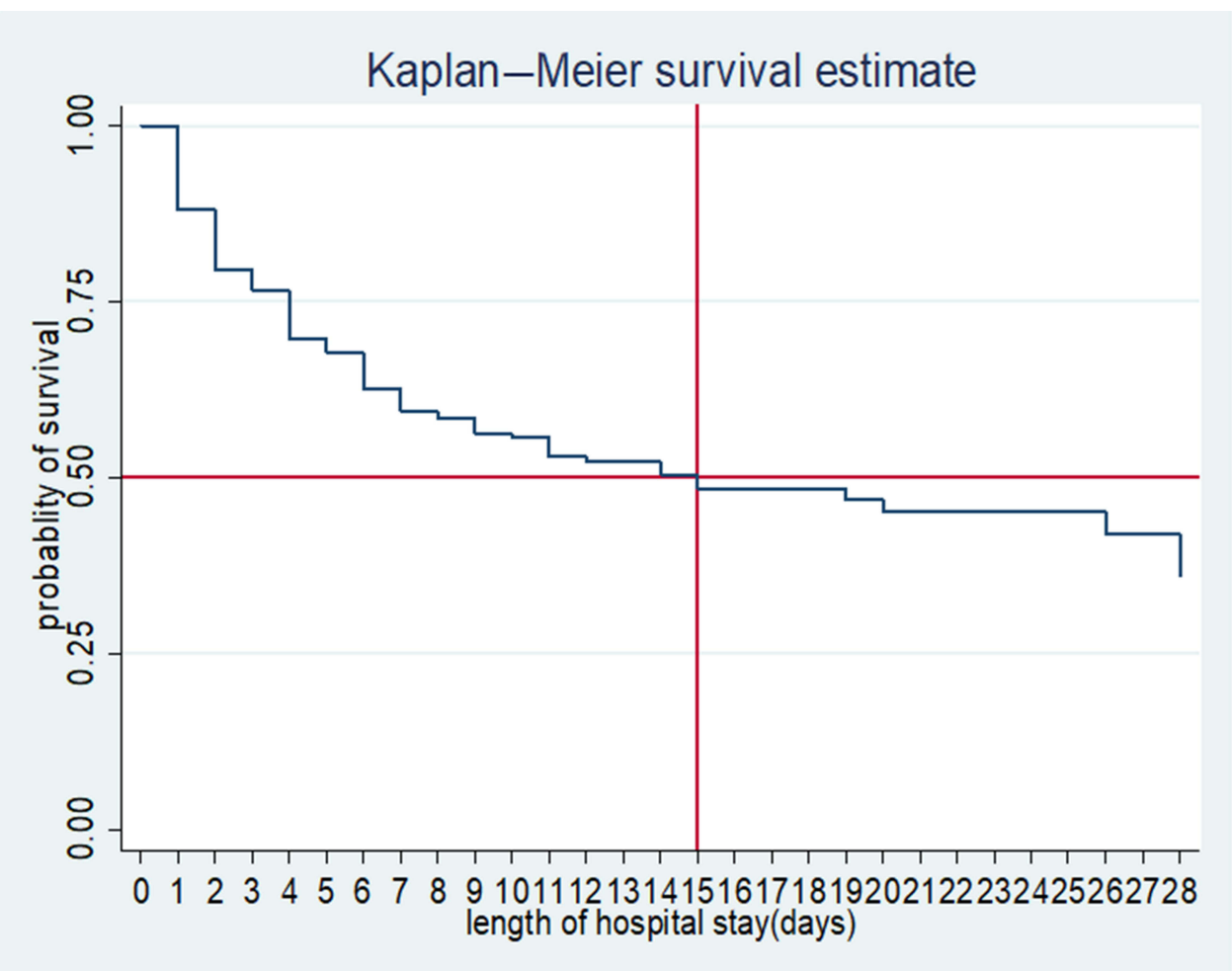

Figure I Kaplan-Meier survival estimate of graph of overall median survival time among preterm neonates at NICU of MTUTH, 2020 (N=568). Comparison of mortality rate and mortality free survival among preterm neonates.

done at the Teaching Hospitals of Iran where $76.9 \%$ of neonates died. ${ }^{18}$ This difference might be due to the difference in gestational age (GA) of study subjects. The study subjects in Iran involved extremely preterm neonates while this study did not have data on extremely preterm neonates. Mortality becomes lower as GA of neonates is increased. This can be supported, in this study, the lower the GA, the more they died.

Generally, this study revealed that the overall probability of survival of preterm neonates at the end period of follow-up was $31.32 \%$ (95\%CI: 14.29-50.02). This may be due to the inverse relation of survival and failure function. This result was in agreement with a study conducted in Gondar, Ethiopia which indicated that $30.62 \%$ survived at the end of the neonatal period. ${ }^{15}$

This study also identified predictors of preterm neonatal mortality. Accordingly, preterm neonates diagnosed with respiratory distress syndrome (RDS) increased the chance of preterm mortality by 1.74 times compared to their counterparts (adjusted hazard ratio, AHR, 1.74, 95\%CI: 1.28-2.36). This finding is supported by the study done in Nigeria, ${ }^{19}$ Jimma University Hospital, ${ }^{14}$ and Tikur Anbessa Hospital. ${ }^{12}$ The possible reason might be the fact that preterm neonates develop RDS due to prematurity-related surfactant deficiency mostly have risk of lung collapse leading to hypoxia finally may end up with death. In this study, preterm neonates who had a fifth-minute APGAR score of $<7$ were 1.87 times more likely at risk of dying than those who had an APGAR score of $>7$ (AHR: 1.87, 95\%CI: 1.31-2.68). This result is supported by other studies conducted in China, ${ }^{20}$ Brazil, ${ }^{21}$ Iran,${ }^{16}$ University of Gondar Hospital, ${ }^{13}$ and Tikur Anbessa Hospital. ${ }^{12}$ This might be due to neonates who experienced the fifth-minute APGAR score of $<7$ are those who need close and critical care and support. Unless they get advanced care by skilled care providers with adequate and appropriate supply of medical equipment, they are more vulnerable to death compared to their counterparts. In this study, preterm neonates diagnosed with hypothermia had 1.36 times hazard of mortality compared to their counterparts (AHR: 1.36, 95\%CI: 1-1.84). This result was supported by the study conducted at Jimma University Hospital. ${ }^{14}$ This might be due to the fact that premature neonates are born with less adipose tissue which predisposes them to risk of complications of hypothermia that can lead to death. In the study hospital there was no adequate incubator which may also increase the risk of death. The hazard of mortality was 2.67 times higher for preterm neonates born with VLBW (1000-1499 g) as compared to those neonates with birth 
Table 4 Results of the Bivariate and Multivariable Analysis of Preterm Neonates at NICU of MTUTH, 2020 (N=568)

\begin{tabular}{|c|c|c|c|c|c|c|}
\hline Covariates & Category & Censored & Death & CHR (95\%Cl) & AHR (95\%Cl) & $P>|z|$ \\
\hline \multirow[t]{2}{*}{ Sex } & Female & 189 & 91 & 1 & 1 & \\
\hline & Male & 180 & 108 & $1.25(0.95-1.66)$ & I.I8 (0.88-I.58) & 0.27 \\
\hline \multirow[t]{2}{*}{ Maternal residency } & Urban & 135 & 43 & I & I & \\
\hline & Rural & 234 & 156 & $1.68(1.20-2.36)$ & $1.29(0.88-1.88)$ & 0.19 \\
\hline \multirow[t]{2}{*}{ Maternal febrile illness } & No & 351 & 179 & I & 1 & \\
\hline & Yes & 18 & 20 & $1.68(1.05-2.67)$ & $1.58(0.94-2.66)$ & 0.085 \\
\hline \multirow[t]{2}{*}{ Type of pregnancy } & Single & 295 & 138 & I & I & \\
\hline & Multiple & 74 & 61 & $1.26(0.93-1.7 I)$ & $0.82(0.59-1.14)$ & 0.245 \\
\hline \multirow[t]{2}{*}{ Antenatal care } & Yes & 342 & 173 & I & 1 & \\
\hline & No & 27 & 26 & $1.88(1.24-2.84)$ & $1.9(1.29-3.01)$ & $0.006 *$ \\
\hline \multirow[t]{2}{*}{ Mother's HIV status } & Negative & 365 & 186 & I & $\mathrm{I}$ & \\
\hline & Positive & 4 & 13 & $2.76(1.57-4.84)$ & $1.6(0.9-2.95)$ & 0.103 \\
\hline \multirow[t]{2}{*}{ Presentation } & Cephalic & 352 & 180 & I & I & \\
\hline & Non-cephalic & 17 & 19 & $1.35(0.84-2.17)$ & $1.8(1.04-3.06)$ & $0.036 *$ \\
\hline \multirow[t]{2}{*}{ RDS } & No & 273 & 99 & I & 1 & \\
\hline & Yes & 96 & 100 & $1.83(1.39-2.42)$ & $1.74(1.28-2.36)$ & $0.000 *$ \\
\hline \multirow[t]{2}{*}{ Necrotizing enterocolitis } & No & 367 & 189 & I & I & \\
\hline & Yes & 2 & 10 & $2.26(1.19-4.27)$ & $\mathrm{I} .7(0.6-2.3 \mathrm{I})$ & 0.65 \\
\hline \multirow[t]{2}{*}{ Asphyxia } & No & 324 & 158 & I & I & \\
\hline & Yes & 45 & 41 & $1.72(1.22-2.42)$ & $1.33(0.91-1.96)$ & 0.14 \\
\hline \multirow[t]{2}{*}{ Jaundice } & No & $320^{\circ}$ & 184 & I & I & \\
\hline & Yes & 49 & 15 & $0.57(0.33-0.96)$ & $0.78(0.45-1.38)$ & 0.40 \\
\hline \multirow[t]{2}{*}{ Hypothermia } & No & 175 & 82 & 1 & 1 & \\
\hline & Yes & 194 & 117 & $1.22(0.92-1.62)$ & $1.36(1-1.84)$ & $0.049 *$ \\
\hline \multirow[t]{2}{*}{ KMC applied } & Yes & 211 & 75 & 1 & I & \\
\hline & No & 158 & 124 & $1.91(1.43-2.55)$ & $1.45(1.06-1.98)$ & $0.020 *$ \\
\hline \multirow[t]{2}{*}{ Mode of delivery } & Cesarean & 49 & 22 & 1 & I & \\
\hline & Vaginal & 320 & 177 & $1.46(0.93-2.27)$ & $1.78(1.05-3.08)$ & $0.031 *$ \\
\hline \multirow[t]{2}{*}{ Breathing status at birth } & Cry at birth & 308 & 77 & I & I & \\
\hline & Did not cry & 61 & 122 & $3.98(2.99-5.30)$ & $2.81(2.03-3.9)$ & $0.000 *$ \\
\hline \multirow[t]{3}{*}{ Weight for gestational age } & AGA & 297 & 129 & 1 & I & \\
\hline & LGA & 17 & 4 & $0.65(0.24-1.76)$ & I.I (0.4-3.08) & 0.85 \\
\hline & SGA & 55 & 66 & $1.99(1.48-2.68)$ & $1.27(0.89-1.8 \mathrm{I})$ & 0.18 \\
\hline
\end{tabular}

(Continued) 
Table 4 (Continued).

\begin{tabular}{|c|c|c|c|c|c|c|}
\hline Covariates & Category & Censored & Death & CHR (95\%Cl) & AHR (95\%Cl) & $P>|z|$ \\
\hline \multirow[t]{2}{*}{ Gestational age } & $28-32$ & 37 & 53 & $1.95(1.4-2.67)$ & 1.01 (0.729-I.44) & 0.955 \\
\hline & $32-36$ & 332 & 146 & 1 & I & \\
\hline \multirow[t]{4}{*}{ Weight } & $<1000$ & 2 & 4 & $3.83(1.22-12.04)$ & I.88 (0.52-6.76) & 0.33 \\
\hline & $1000-1499$ & 40 & 61 & $3.81(2-7.22)$ & $2.67(1.29-5.53)$ & $0.008^{*}$ \\
\hline & $1500-2499$ & 264 & 123 & $2.21(1.18-4.07)$ & $2.24(1.15-4.39)$ & $0.02 *$ \\
\hline & $\geq 2500$ & 63 & 11 & 1 & I & \\
\hline \multirow[t]{2}{*}{ APGAR at Ist minute } & $<7$ & 247 & 162 & 1.67 (I.I7-2.38) & $0.74(0.47-1.16)$ & 0.191 \\
\hline & $\geq 7$ & 122 & 37 & I & I & \\
\hline \multirow[t]{2}{*}{ APGAR 5th minute } & $<7$ & 98 & 116 & $2.44(1.84-3.24)$ & I.87(I.3I-2.68) & $0.001 *$ \\
\hline & $\geq 7$ & 271 & 83 & I & I & \\
\hline
\end{tabular}

Notes: *Significant at $P$-value $<0.05$ in multivariable analysis. $I=$ considered as reference category.

weight $\geq 2500$ g (AHR: $2.67,95 \%$ CI: $1.29-5.53$ ). This result was supported by the study done in Iran. ${ }^{16}$ This might be due to the fact that VLBW and LBW neonates have high body surface area to body weight ratio and less body brown fat that predispose to hypothermia and hypoglycemia which will further leads to death. Preterm neonates who did not receive KMC were 1.45 times more likely vulnerable to death than their counterparts (AHR: 1.45, 95\%CI: 1.06-1.98). This study was in line with the study done at University of Gondar Hospital. ${ }^{13}$ This might be because neonates who did not receive KMC service are at risk of hypothermia, inability to breathe and delaying of initiation of breastfeeding. These may lead to preterm complications of hypoglycemia and severe hypothermia which may result in death. The hazard of mortality for preterm neonates who did not cry immediately after birth was 2.81 times higher compared to neonates who cried immediately (AHR: $2.81,95 \%$ CI: 2.03 3.93). This finding was supported by the study done at University of Gondar hospital. ${ }^{13}$ This might be due to the fact that preterm neonates who did not cry immediately may show disturbed breathing effect and newborn reflexes are not normal that indirectly indicates risk for asphyxia that can lead to death. In addition, preterm neonates who were born from mother who did not have ANC follow-up had 1.9 times higher hazard of death compared to their counterparts (AHR: 1.9, 95\%CI: 1.29-3.01). This study finding was supported by the study done at the University of Gondar Hospital. ${ }^{15}$ The possible reason might be lack of ANC visits which may result in inadequate monitoring of pregnancy and might lead to neonatal complications during and after delivery which may be associated with increased risk of neonatal death. Furthermore, in this study, preterm neonates who were born in non-cephalic presentation had 1.8 times higher hazard of mortality than their counterparts (AHR: 1.8, 95\% CI: 1.04-3.06). This was supported by the study conducted in Iran. ${ }^{16}$ This might be due to the fact that preterm neonates delivered with non-cephalic presentation in early gestational age had a greater risk for asphyxia and meconium aspiration syndrome that further leads to complication and death. Moreover, this study indicated that vaginal mode of delivery had 1.78 times higher hazard of mortality compared to cesarean section (AHR: 1.78, 95\%CI: 1.05-3.08). This result was supported by studies conducted in $\operatorname{Japan}^{22}$ and a global and multi-country survey. ${ }^{23}$ This might be due to the fact that vaginal delivery may result in a stressful or traumatic birth and prolonged labor process that exposes the risk of stress and relative hypoxia and other complications which may lead to death.

\section{Limitation of the Study}

Since it is retrospective study, the study did not address the probable service-related predictors of mortality.

\section{Conclusion}

In conclusion, this study revealed that incidence rate and the proportion of preterm neonatal mortality was high. Since almost all of the identified predictors are preventable and treatable, this calls everyone to work toward prevention, 
early anticipation, and management of these predictors. Therefore, due attention toward prevention and early management of these predictors should be emphasized by health-care providers and hospital administration. Furthermore, researchers should investigate health-service related predictors of premature mortality prospectively.

\section{Abbreviations}

AHR, adjusted hazard ratio; ANC, antenatal care; APGAR, appearance, pulse, grimace, activity, respiration; GA, gestational age; HIV, human immunodeficiency virus; $\mathrm{HR}$, hazard ratio; KMC, kangaroo mother care; LBW, low birth weight; MTUTH, Mizan Tepi University Teaching Hospital; NICU, Neonatal Intensive Care Unit; RDS, respiratory distress syndrome.

\section{Acknowledgments}

We would like to thank Jimma University for financial support of this study. We would also like to acknowledge the hospital administrators for their cooperation and support in the success of this study. Our deepest gratitude is also extended to instructors at the school of Nursing and Midwifery of Jimma University who supported us either directly or indirectly by giving a constructive comment and guidance. Last but not least, we could not wrap up without thanking our dedicated collectors for their support and encouragement throughout this work.

\section{Funding}

Jimma University covered the survey cost and there is no additional fund for this particular study.

\section{Disclosure}

The authors report no conflicts of interest in this work.

\section{References}

1. Phelan L, Harris J, Smith C, Look V, Ring S. Nutrition practice care guidelines for preterm infants in the community. 2016.

2. Bieleninik $€$, Gold C. Early intervention for premature infants in neonatal intensive care unit. Acta Neuropsychologica. 2014;12 (2): 185-203.

3. Ashish KC, Wrammert J, Nelin V, Ewald U, Clark R, Malqvist M. Level of mortality risk for babies born preterm or with a small weight $\mathrm{p}$ for gestation in tertiary hospital of Nepal. BMC Public Health.2015;15(1):1-9.

4. United Nations Inter-agency Group for Child Mortality Estimation (UN IGME). Levels \& Trends in Child Mortality: Report 2019, Estimates Developed by the United Nations Inter-Agency Group for Child Mortality Estimation. United Nations Children's Fund; 2019.
5. Sankar MJ, Natarajan CK, Das RR, Agarwal R, Chandrasekaran A, Paul VK. When do newborns die? A systematic review of timing of overall and cause-specific neonatal deaths in developing countries. $J$ Perinatol. 2016;36:S1-S11. doi:10.1038/jp.2016.27

6. Ethiopian Public Health Institute (EPHI) [Ethiopia] and ICF. 2019. Ethiopia Mini Demographic and Health Survey 2019: Key Indicators. Rockville, Maryland, USA: EPHI and ICF. Available from: https:// dhsprogram.com/pubs/pdf/FR363/FR363.pdf. Accessed August 29, 2021.

7. Directoratefederal $\mathrm{CH}$. National strategy for newborn and child survival in Ethiopia national strategy for newborn and child survival in Ethiopia. 2019. June 2015.

8. Mengesha HG, Wuneh AD, Lerebo WT, Tekle TH. Survival of neonates and predictors of their mortality in Tigray region, Northern Ethiopia: prospective cohort study. BMC Pregnancy Childbirth . 2016;16(December):1-13. doi:10.1186/s12884-0160994-9

9. Desalew A, Sintayehu Y, Teferi N, Amare F, Geda B, Worku T. Cause and predictors of neonatal mortality among neonates admitted to neonatal intensive care units of public hospitals in eastern Ethiopia: a facility-based prospective follow-up study. $B M C$ Pediatr. 2020;20:1-11.

10. Mekonnen T, Tenu T, Aklilu AT, Abera T. Assessment of neonatal death and causes among admitted neonates in neonatal intensive care unit of Mizan Tepi University Teaching Hospital, Bench Maji Zone, South-West Ethiopia, 2018. Clin Mother Child Health. 2018;15(4):2. doi: $10.4172 / 2090-7214.1000305$

11. EWE Child. The global strategy for women's children's and adolescents' health (2016-2030):. I at glance.2015. STRATEGY. 2016.

12. Asmare Y. Survival status and predictor of mortality among premature neonate that was admitted to neonatal intensive care unit from 2013-2017 at Tikur Anbessa Hospital, Addis Ababa Ethiopia: conference presentation. Addis Ababa Univ. 2018;8:4172.

13. Yismaw AE, Gelagay AA, Sisay MM. Survival and predictors among preterm neonates admitted at University of Gondar comprehensive specialized hospital neonatal intensive care unit, Northwest Ethiopia. Ital J Pediatr. 2019;45(1):1. doi:10.1186/s13052-018-0597-3

14. Wesenu M, Kulkarni S, Tilahun T. Modeling determinants of time-todeath in premature infants admitted to neonatal intensive care unit in Jimma University Specialized Hospital. Ann Data Sci. 2017;4 (3):361-381. doi:10.1007/s40745-017-0107-2

15. Yehuala $S$, Teka Z. Survival analysis of premature infants admitted to neonatal intensive care unit (NICU) in Northwest Ethiopia using semi-parametric frailty model. J Biometr Biostat. 2015;6(1):1-12.

16. Haghighi L, Nojomi M, Mohabbatian B, Najmi Z. Survival predictors of preterm neonates: hospital based study in Iran (2010-2011). Iran J Reprod Med. 2013;11(12):957-964.

17. Khan MR, Maheshwari PK, Shamim H, Ahmed S, Ali SR. Morbidity pattern of sick hospitalized preterm infants in Karachi, Pakistan. J Pak Med Assoc. 2012;62(4):386.

18. Saboute M, Kashaki M, Bordbar A, Khalessi N, Farahani Z. The incidence of respiratory distress syndrome among preterm infants admitted to neonatal intensive care unit: a retrospective study. Open J Pediatr. 2015;5:285-289.

19. Fajolu I, Akintan PE, Ezenwa B, Ezeaka VC. Survival of extremely preterm neonates in a resource- limited setting. Iran J Neonatol. 2019;10(3):40-46.

20. Kong X, Xu F, Wu R, et al. Neonatal mortality and morbidity among infants between 24 to 31 complete weeks: a multicenter survey in China from 2013 to 2014. BMC Pediatr. 2016;16:1-8. doi:10.1186/ s12887-016-0716-5.

21. Campos E, De CM, Jorge Á, et al. Perinatal factors associated with early neonatal deaths in very low birth weight preterm infants in Northeast Brazil. BMC Pediatr. 2014;14:1-8. 
22. Ancel PY, Goffinet F, Kuhn P, et al. Survival and morbidity of preterm children born at 22 through 34 weeks' gestation in France in 2011 results of the EPIPAGE-2 cohort study. JAMA Pediatr. 2015;169(3):230-238. doi:10.1001/jamapediatrics.2014.3351
23. Thanh BYL, Lumbiganon P, Pattanittum P, et al. Mode of delivery and pregnancy outcomes in preterm birth: a secondary analysis of the WHO global and multi-country surveys. Sci Rep. 2019;9(1):1-8. doi:10.1038/s41598-019-52015-w

\section{Publish your work in this journal}

Pediatric Health, Medicine and Therapeutics is an international, peerreviewed, open access journal publishing original research, reports, editorials, reviews and commentaries. All aspects of health maintenance, preventative measures and disease treatment interventions are addressed within the journal. Practitioners from all disciplines are invited to submit their work as well as healthcare researchers and patient support groups. The manuscript management system is completely online and includes a very quick and fair peer-review system. Visit http://www.dovepress.com/testimonials.php to read real quotes from published authors. 